\title{
Lidil
}

Revue de linguistique et de didactique des langues

$44 \mid 2011$

Langues, minor(is)ations et marginalisations

\section{Dynamiques sociolinguistiques dans une communauté plurilingue : des lycéens éthiopiens entre conformisme et émancipation}

\section{Véronique Miguel Addisu}

\section{OpenEdition}

\section{Journals}

Édition électronique

URL : http://journals.openedition.org/lidil/3143

DOI : $10.4000 /$ lidil.3143

ISSN : 1960-6052

Éditeur

UGA Éditions/Université Grenoble Alpes

\section{Édition imprimée}

Date de publication : 15 décembre 2011

Pagination : $93-110$

ISBN : 978-2-84310-212-7

ISSN : $1146-6480$

Référence électronique

Véronique Miguel Addisu, «Dynamiques sociolinguistiques dans une communauté plurilingue : des lycéens éthiopiens entre conformisme et émancipation », Lidil [En ligne], 44 | 2011, mis en ligne le 15 juin 2013, consulté le 30 avril 2019. URL : http://journals.openedition.org/lidil/3143 ; DOI : 10.4000/ lidil.3143 


\title{
Dynamiques sociolinguistiques dans une communauté plurilingue : des lycéens éthiopiens entre conformisme et émancipation
}

\author{
Véronique Miguel Addisu*
}

\begin{abstract}
RÉSUMÉ
Lorsque langues et cultures sont en contact, les normes sociolinguistiques des locuteurs révèlent la force des enjeux identitaires. L'analyse de discours d'élèves adolescents de plusieurs nationalités étudiant tous en français dans un contexte plurilingue (l'Éthiopie) montrera comment ils créent une synergie entre norme exogène (du français) et norme endogène (le parler bilingue), se disant à la fois semblables et différents parmi les pairs.
\end{abstract}

\begin{abstract}
When languages and cultures are in contact, interlocutors' sociolinguistic norms reveal the extent to which identity is at stake. A discourse analysis of adolescent pupils of various nationalities studying in French in a multilingual context (Ethiopia) will show how they create synergy between a (French) exogenous norm and a (bilingual) endogenous norm, presenting themselves both as similar to their peers and distinct from them.
\end{abstract}

L'étude sociolinguistique de terrains plurilingues actualise les concepts de norme sociolinguistique et de domination linguistique : les pratiques et représentations langagières en circulation dans les communautés plurilingues révèlent des dynamiques peu visibles dans un milieu unilingue. La première spécificité de ces terrains plurilingues renvoie aux types de données linguistiques convoquées : le fait que plusieurs langues soient parlées rend les variations plus facilement observables pour le chercheur

* Université de Rouen, laboratoire LiDiFra. 
mais leur pluralité gêne fortement les dénominations linguistiques telles que «le » français ou «le » parler bilingue ${ }^{1}$ :

Aucune langue ne se présente comme un ensemble unique de règles. Toutes connaissent de multiples variétés ou lectes, dont la diversité est masquée par des étiquettes au singulier (LE français, LE turc, etc.). Le caractère commode de ces dénominations ne doit cependant pas masquer leur caractère abstrait et réducteur. (Moreau, 1997 : 283-284)

Ces terrains ont aussi des frontières éminemment floues et pourtant géographiquement définies : il est commode d'isoler une « communauté linguistique » mais le linguistique est social parce qu'il dépend des acteurs, ce qui signifie que c'est avant tout une communauté sociale. En effet, « l'objet d'étude de la linguistique n'est pas seulement la langue ou les langues, mais la communauté sociale sous son aspect linguistique » (Calvet, 1994 : 116). Dans de nombreuses situations, un mode d'expression parmi d'autres s'impose comme seul légitime, à condition «que le marché linguistique soit unifié et que les différents dialectes (de classe, de région ou d'ethnie) soient pratiquement mesurés à la langue ou à l'usage légitime» (Bourdieu, $1982: 28$ ).

Mais comment identifier «l'usage légitime» ? Les données recueillies seront différentes selon les contours que le chercheur donne à la communauté qu'il étudie. Or « les normes endogènes ne peuvent être envisagées indépendamment des normes exogènes, ni les normes linguistiques en dehors des normes sociales » (Bavoux, Prudent, Warthon, 2008 : 13). C'est ce lien que je propose d'étudier à partir de pratiques et représentations langagières d'élèves adolescents de nationalités diverses apprenant tous en français au Lycée Guebre Mariam (désormais LGM), établissement scolaire de l'AEFE ${ }^{2}$ à Addis-Abeba (Éthiopie), lieu de ma recherche doctorale menée principalement sur le terrain de 2005 à 2010. Comment l'appropriation des règles linguistiques du français langue de scolarisation participe-t-elle à la construction identitaire dans un groupe plurilingue et pluriethnique qui revendique un parler métissé?

Après avoir précisé ce que la notion de norme recouvre dans l'appréhension sociolinguistique des terrains plurilingues, je rendrai compte de la valeur attribuée aux parlers métissés par les élèves du LGM. Je croiserai pour cela plusieurs types de discours, les pratiques effectives

1. Voir par exemple les questions soulevées par Berhnard Pöll et Elmar Schafroth (2009) et par Henri Boyer (2010) pour la notion d' « hybridation linguistique ».

2. Agence pour l'Enseignement Français à l'Étranger. 
observées, des tendances attestées dans 124 questionnaires et des entretiens semi-dirigés menés avec 29 élèves. Bien qu'ils revendiquent le fait que le français ne soit qu'une langue parmi d'autres dans la pluralité des répertoires utilisés, l'importance qu'ils accordent à la « faute » en français entre pairs révèle d'autres processus normatifs qui participent aux rapports de pouvoir à l'intérieur même de la communauté. Nous verrons alors comment les sanctions - fréquentes - influent sur les positionnements identitaires de chacun :

Dans la mesure où l'existence des groupes ethniques dépend de l'entretien de leurs frontières, la question est de savoir comment les dichotomisations entre membres et outsiders sont produites et maintenues et de discerner leur effet propre sur les comportements effectifs. » (Poutignat et Streiff-Fenart, 2005 [1995] : 123)

Associée à une norme endogène plurilingue revendiquée comme une contre-norme, la norme exogène est mobilisée à des fins identitaires ; ces dynamiques sont fondées à la fois sur la différenciation par le français et sur l'unification par les pratiques métissées.

\section{Quelles règles? Quelles normes? Pour qui?}

\section{Vers une définition de la norme en sociolinguistique}

Alain Rey souligne la différence conceptuelle entre deux acceptions homonymiques du terme «norme » : l'une qui réfère à l'observation des pratiques et l'autre qui renvoie à un système de valeurs, « un faisceau d'intentions subjectives » (Rey, 1972 : 5). Cette partition permet d'éviter de confondre description et prescription dans le discours commun et renvoie à l'analyse pragmatique des règles qui président aux interactions verbales. Searle distingue ainsi les règles régulatives ${ }^{3}$ des règles constitutives. Les premières «ont pour fonction de régir une activité préexistante, une activité dont l'existence est logiquement indépendante des règles »; les deuxièmes « créent ou définissent de nouvelles formes de comportement » (Searle, 1972 : 72-73). L'auteur prend l'exemple des règles du football : c'est parce qu'elles existent que le jeu existe. Ce sont donc des règles constitutives, à la différence des règles de politesse, qui

3. L'auteur utilise de façon indifférenciée les termes « régulatif» et «normatif». Ce ne sera pas le cas ici, comme on le comprendra plus bas. 
sont régulatives puisqu'elles régissent une activité préexistant à la règle (le respect de l'autre). Les langues sont donc conventionnelles (Searle, 1972 : 77-78) et les linguistes les étudient comme telles.

Lorsque, dans le champ social, on s'approprie et diffuse ces règles comme des règles nécessaires, elles deviennent légitimes et régulent les interactions verbales parce que celles-ci sont aussi sociales. C'est parce que ceux qui y jouent lui donnent le sens de «football » que les règles de ce jeu sont effectivement légitimes pour le spécifier. Appliquées au français, quand des règles constitutives sont reconnues comme définitoires de l'objet « français » par les locuteurs, elles peuvent devenir des normes prescriptives. Les représentations (sociales) du même objet linguistique peuvent d'ailleurs largement différer. Les données recueillies dans les interactions sont plutôt des traces de dynamiques sociolinguistiques, autrement dit, le produit complexe des forces sociales et linguistiques. William Labov (1976) est sans doute le premier à avoir montré que les «manières de parler » sont sujettes à des évaluations sociales complexes : le locuteur ne parle pas toujours comme il le dit, la variation linguistique est marquée par des influences externes, sociales et systémiques. La sociolinguistique vise donc à révéler ces processus normatifs, particulièrement saillants sur les terrains plurilingues. Ainsi, prenant l'exemple des dynamiques du français en Côte d'Ivoire, Nicolaï montre l'intérêt des catégorisations searliennes tout en les nuançant et relève que l'on peut se situer à l'extérieur du groupe de ceux qui reconnaissent ces règles comme constitutives; dans ce cas, les règles de la langue dominante (ici le français) ne sont pas reconnues comme telles et ne régulent donc plus le contenu des interactions, mais les actualisent. On peut ainsi créer une autre langue ou même attribuer au français d'autres règles dites constitutives (Nicolaï, 2011 : 47). Au sein d'une école française, les règles normatives sont légitimées du fait qu'elles sont identifiées comme « constitutives » par les programmes, les locuteurs natifs, etc. Il faut «bien parler français » pour « réussir à l'école». Parce qu'elles sont conventionnelles, ces règles sont aussi des enjeux sociaux. Elles ont ainsi au moins deux propriétés complémentaires : elles permettent au système d'exister, mais elles permettent aussi à ceux qui utilisent ce système de se constituer en tant que groupe, notamment par le biais de normes prescriptives. Je retiendrai donc ici qu'une norme sociolinguistique est une règle langagière considérée pour sa portée sociale et identitaire. La norme prescriptive en est un cas particulier, en tant que règle appliquée avec un but explicite de régulation des comportements sociaux. 
Les normes prescriptives, en s'appliquant au système linguistique, peuvent le transformer tout en étant aussi un critère d'évaluation sociale. Mettre au jour la fréquence et la portée des injonctions normatives portant sur le «bien dire » dans un groupe rend donc compte des idéologies langagières en circulation, ce que révèle aussi la force des sanctions afférant à leur transgression.

Étudier les dynamiques normatives en circulation pour un groupe de locuteurs revient à faire émerger les catégorisations pratiques des locuteurs dans leurs discours. Dans un contexte scolaire diglossique tel que le LGM, la notion largement diffusée de « faute » en français parait particulièrement pertinente pour comprendre comment les jeunes s'approprient les règles du français en tant qu'outil de pouvoir et de distinction.

\section{Dynamiques normatives et idéologies}

Les idéologies linguistiques génèrent des normes prescriptives (notamment par le biais de l'école) qui sont, dans les sociétés dites monolingues, largement diffusées par les travaux de standardisation de la langue dominante :

Les idéologies linguistiques ne concernent ainsi pas la langue seulement, mais plutôt différentes visions des liens entre les langues et l'identité (par exemple nationale), qui s'actualisent par le biais, notamment, des modèles de socialisation prévalents, des rituels religieux, de l'école, etc.

(Moore et Py, 2008 : 274)

Dans les sociétés plurilingues, il est fréquent que l'on ait cherché à standardiser une seule langue, notamment par le développement de normes prescriptives. Celle-ci devient la langue dominante, et la langue des enseignements ${ }^{4}$. Les enjeux politiques et identitaires qui y sont liés révèlent la subjectivité des normes évaluatives : un groupe peut estimer qu'il est « normal » d'user d'une forme linguistique, en se positionnant en accord (ou en désaccord) avec la norme prescriptive. Ainsi, tous les élèves du LGM utilisent fréquemment des interjections éthiopiennes

4. On pense ici à nombre de pays africains francophones, mais aussi à l'Éthiopie : c'est l'amharique qui a été standardisé au XIX ${ }^{\mathrm{e}}$ siècle puis enseigné, même si la politique linguistique actuelle vise à développer la standardisation de plusieurs autres langues éthiopiennes. 
entre pairs ( «baka ${ }^{5} », \ll \operatorname{erreh}^{6} » \ldots$ ) mais ne le font pas en classe, montrant ainsi des positionnements normatifs différents selon les espaces scolaires.

Les normes sont l'expression de valeurs, liens notamment étudiés en sociologie : la (non) adhésion à une norme dominante (dans les attitudes et le comportement) met en évidence les valeurs de l'acteur social, qui prend le risque de la sanction assortie à la transgression d'une norme dominante (Demeulenaere, 2003). La sanction peut être interne (la culpabilité) et/ou externe (la pression sociale). Si les sanctions externes sont faibles, le sentiment de solidarité des individus ayant un même comportement se renforce et pousse à des transgressions plus nombreuses. Lorsque la sanction est forte, la déviance est d'autant plus couteuse. S'agissant du langage, l'insécurité linguistique est un signe fort de la peur de la sanction. Mais dans le même temps, plus la valeur associée à la transgression est forte pour l'individu, plus il sera enclin à dévier de la norme dominante. Ainsi, les attitudes et comportements non conformes, lorsqu'ils sont largement partagés dans un groupe, disent quelque chose de valeurs sous-jacentes au groupe et non reconnues par les dominants. Ils disent aussi quelque chose de la cohésion du groupe et du sentiment de solidarité de ses membres : la solidarité renforce le prestige de la valeur partagée.

Au LGM, école française implantée dans un pays plurilingue, le parler métissé est une norme endogène à la communauté des élèves, associée à des valeurs fortes de solidarité et d'identité. Pourtant, les élèves sanctionnent eux-mêmes leurs «fautes » en français. Cela dessine une communauté linguistique aux contours complexes : en assignant plusieurs statuts différents à une même langue, les identités adolescentes se construisent de façon différenciée à l'intérieur d'un système normatif caractérisé par la tension entre norme endogène et norme exogène. La notion de « communauté linguistique » devient alors un outil heuristique pertinent parce qu'au lieu de gommer la pluralité des normes, elle en révèle les processus d'interaction. Nous allons ainsi étudier comment la norme du parler multilingue s'articule avec celle du «bien dire » en français.

5. Traduction : «ça suffit ».
6. Traduction : «c'est pas vrai! » 


\section{Les normes langagières entre adolescents}

Le LGM scolarise plus de 1700 élèves, dont environ 800 au secondaire. En 2007, $64 \%$ des élèves du secondaire sont éthiopiens et $10 \%$ sont français, plus de trente nationalités sont représentées (plus de $20 \%$ sont africains francophones). C'est un terrain diglossique à double titre : diglossie sociale et diglossie scolaire. Les élèves utilisent plusieurs langues pour communiquer entre pairs, ce qui est valorisé par l'environnement social : l'Éthiopie est un État fédéral fondé sur des bases ethnolinguistiques. Seuls les élèves éthiopiens ont des cours d'amharique, tous se préparent au baccalauréat et suivent des enseignements en français, sur le mode unilingue : l'alternance codique n'est pas tolérée dans la classe ou dans les interactions avec l'équipe de direction. Notre corpus a été recueilli auprès de 124 élèves adolescents de nationalités différentes ${ }^{7}$ vivant dans une ville plurilingue et déclarant parler plusieurs langues en famille (82\%). Leurs répertoires verbaux sont largement asymétriques, même s'ils ont tous une langue en commun : le français. La norme dominante pour les interactions avec les adultes dans l'établissement est l'unilinguisme (98\% des élèves n'utilisent que le français avec les enseignants français) mais c'est le mélange des langues qui est valorisé entre pairs $(77,4 \%$ usent d'un parler métissé entre pairs).

\section{Une norme endogène : prestige de la pluralité}

Une forte valeur de prestige est associée à ces pratiques plurilingues érigées en « parler jeune ${ }^{8}$ ». Ces revendications sont largement attestées entre pairs : « on se sent libre », « on ne parle pas comme avec les adultes ${ }^{9} »$, etc. On utilise plusieurs langues dont les principales sont le français, l'amharique et l'anglais. Dans les questionnaires, $72 \%$ estiment qu'il est « naturel » de mélanger les langues et dans 27 entretiens ce mélange est érigé en valeur : selon eux, cela montre que l'on est

7. Le questionnaire, rempli en 2006 et en 2008 par un échantillon représentatif de 124 élèves comportait les rubriques suivantes : toi et les langues, toi et l'apprentissage, francophonie/bilinguisme/scolarité, les cultures, toi et les adultes. Ils ont été suivis par 29 entretiens d'explicitation des questionnaires avec 29 élèves volontaires. Les pratiques déclarées ont été comparées aux pratiques réelles.

8. On retrouve des pratiques et revendications similaires dans d'autres contextes scolaires, voir par exemple les travaux de Monica Heller (2003) et de Ben Rampton (2004).

9. Extraits récurrents dans les entretiens. 
tolérant et que l'on respecte la diversité des appartenances culturelles, on renforce ainsi la solidarité du groupe en se forgeant une part identitaire commune ${ }^{10}$ :

ÉLISABETH : [...] (sourire) dans les cours c'est que le français et dans la cour je mélange tout l'temps / dans la classe je dois m'exprimer d'une façon + je trouve que + le prof doit me comprendre / dans la cour + je mélange hors de la classe pour + je sais + mes amis vont me comprendre !

Plus pragmatiquement, l'intérêt majeur de ce type de communication est que l'on peut s'exprimer et comprendre même si l'on estime que l'« on ne parle pas bien français ».

Ne pas parler « uniquement» français permet aussi de revendiquer une identité non française : $92 \%$ se disent francophones mais seuls $35 \%$ déclarent avoir au moins un « trait culturel » français ${ }^{11}$, ce qui peut être mis en relation avec le fait que ces adolescents ne considèrent pas le français comme une de leurs langues d'appartenance, alors même qu'ils acquièrent depuis plusieurs années de nouveaux savoirs en français :

Le standard français proposé comme modèle à toute la francophonie n'est pas neutre d'identité : qui, dans la francophonie extra-hexagonale, se conforme strictement à la norme [...] est identifié comme un Français. Et, bien sûr, une telle caractérisation ne peut pas satisfaire les personnes soucieuses à la fois de pratiquer une variété de prestige et de préserver leur identité, tant par rapport à l'endo-groupe que par rapport à l'exo-groupe. En conséquence de quoi elles se conforment à la norme

10. Les élèves cités sont présentés en annexe.

11. Cette expression fait référence à une question du questionnaire libellée ainsi : «On peut considérer que les traits culturels sont ce qui fait l'identité d'une personne qui appartient à un groupe précis. On parle ainsi de la « culture française », de la «culture éthiopienne »... mais aussi de la « culture scolaire », de la « culture familiale »... Si elle appartient à plusieurs groupes, une personne peut évidemment avoir plusieurs cultures en elle. Un trait culturel peut être une manière de manger, une manière de croire, une manière d'imaginer la famille, une manière de penser, une manière de danser... » (partie E). Cette introduction était donc formulée de façon à ne pas réifier les cultures, tout en permettant l'expression de certains stéréotypes le cas échéant. On demandait ensuite si l'adolescent pensait avoir des « trait culturels » éthiopiens, français, autres... Sachant que plus de $90 \%$ d'entre eux étaient scolarisés au LGM depuis l'âge de trois ans et que «l'identité LGM » est reconnue partout en ville comme particulière, on pouvait s'attendre à des réponses positives bien plus nombreuses ici. 
pour l'essentiel [...] mais elles accueillent dans leur langue différents traits grâce auxquels elles affirment leur appartenance à leur groupe : ainsi émergent de nouvelles normes linguistiques, des normes endogènes. (Moreau, $2000: 147$ )

Pour $70 \%$ des locuteurs, cette norme endogène revendiquée n'est pas « une » langue mais plutôt un ensemble de pratiques et de représentations. Le produit de ce processus d'individuation sociolinguistique ${ }^{12}$ pourrait être catégorisé comme un système linguistique (plutôt qu'une langue ${ }^{13}$ ) polynomique :

Une langue polynomique est une langue à l'unité abstraite, à laquelle les utilisateurs reconnaissent plusieurs modalités d'existence, toutes également tolérées sans qu'il y ait entre elles hiérarchisation ou spécialisation des fonctions. Elles s'accompagnent de l'intertolérance entre utilisateurs de variétés différentes sur les plans phonétiques et morphologiques, de même que la multiplicité lexicale est conçue ailleurs comme un élément de richesse. Il s'agit en quelque sorte de langues à norme molle ou souple [...]. (Marcellesi, 2003 [1986] : 173)

Si l'on considère le choix d'une langue plutôt qu'une autre comme une « norme » (c'est-à-dire comme une règle langagière considérée pour sa portée sociale et identitaire), alors elles sont effectivement souples sur notre terrain et illustrent un «plurilinguisme de tolérance », caractérisé par la pluralité des usages ${ }^{14}$.

Pourtant, l'analyse des entretiens montre que si certains disent qu' « on se comprend mieux », d'autres se sentent mal à l'aise, justement parce qu'ils ne comprennent pas tout. Or la notion de « compréhension » peut recouvrir des réalités différentes pour les locuteurs ${ }^{15}$ : Michael (francoéthiopien venant de France) me dit qu'il comprend ce que sa petite amie

12. Marcellesi, 2003 [1986], p. 169. L'individuation sociolinguistique est le processus « par lequel une communauté ou un groupe social tend à systématiser ses différences, à les sacraliser, à les considérer comme déterminantes, à en faire un élément de reconnaissance. Elles deviennent alors des indicateurs d'identité ».

13. En effet, « une langue nommée d'après le nom d'un État-nation paraît aujourd'hui plus réelle qu'une autre. [...] Les "images" des langues varient selon les cultures qui les élaborent et les acceptent, selon les idéologies » (Rey, 2010).

14. Pour une analyse plus développée, voir aussi Véronique Miguel Addisu (2010).

15. Ce que Jean-Baptiste Marcellesi note aussi (2003:27). 
éthiopienne lui dit en amharique alors que celle-ci m'affirme dans l'entretien suivant qu'il ne peut pas comprendre l'amharique et qu'elle lui parle donc en français... Ces normes « molles » - autrement dit le prestige attribué aux pratiques métissées - seraient-elles donc une valorisation du contact de langues pour masquer le conflit entre le «bien comprendre » grâce au français et le «bien s'entendre » grâce aux pratiques communautaires? Celles-ci suffisent-elles à assurer la cohésion du groupe? Pour y répondre, il faut comprendre quelle place a le français dans ces interactions plurilingues. Je vise ici à faire émerger la part d'hégémonie ${ }^{16}$ du français, alors même que les élèves déclarent s'en émanciper. L'objet n'étant pas ici quantitatif (une langue utilisée) mais qualitatif (des normes y afférant), c'est la notion de «faute » que l'on étudiera ici.

\section{Une norme exogène : pouvoir et insécurité}

Les élèves stigmatisent fortement entre pairs la «faute » en français, à l'intérieur même des discours métissés. Les valeurs associées aux sanctions sont apparemment paradoxales : c'est humiliant mais nécessaire. Les évaluations génèrent des conflits d'ordre sociolinguistique.

Une première question a été posée à propos des enseignants dans les questionnaires : «Donne trois exemples de «fautes » en français que les profs reprennent toujours » (Q. B.4) : seuls $61 \%$ des élèves ont répondu (soit 74 sur 124), ce qui parait surprenant du point de vue d'un enseignant qui a l'impression de sans cesse reprendre les formulations « fautives » des élèves. On perçoit ici que le degré d'attention porté à la forme linguistique est différent pour l'enseignant et l'élève. Pourtant $91 \%$ des 74 réponses portent sur le code linguistique. On pourrait alors en conclure que la pression normative en classe est relativement forte et faire l'hypothèse que les élèves s'en émancipent en mélangeant justement les langues en dehors des cours. C'est en tout cas ce dont les réponses attestent lorsqu'on fait émerger les représentations associées

16. «Sauf exception, c'est l'hégémonie qui agit dans les changements sociolinguistiques. On entend par là le processus par lequel les locuteurs des langues dominées sont conduits à considérer comme une bonne chose, allant dans le sens de leurs intérêts matériels et/ou culturels, la prééminence accordée à un système autre que le leur. Contrairement à la domination, l'hégémonie s'accompagne d'une certaine forme de conviction et de consentement. » (Marcellesi, 2003 [1986] : 166). 
au parler métissé entre pairs, ce que mes observations personnelles ont confirmé.

Mais les réponses à la question portant sur les fautes entre pairs ne vont pas dans ce sens ${ }^{17}$ : $60 \%$ des élèves y répondent et la plupart des occurrences relèvent de faits langagiers abondamment sanctionnés en classe. La formulation de la question induisait un positionnement normatif, qui pouvait être compris soit du point de vue des règles interactionnelles entre pairs («fautes [...] qu'il ne faut pas faire entre élèves »), soit du point de vue des règles du français ( « faute en français qu'il ne faut pas faire $[. .] »$.$) . Or, la plupart évoquent comme « fautes » des$ formes linguistiques qui ont toutes en commun d'être à la fois la cause la plus fréquente d'erreurs pour un allophone (le genre, l'emploi des prépositions, les constructions verbales, les pronoms) et des contraintes linguistiques qui ne nuisent que partiellement à l'intelligibilité des discours ${ }^{18}$. Seuls dix élèves évoquent une variation considérée comme non légitime du français, et deux considèrent le mélange des langues comme une « faute » entre pairs. Ces élèves identifient ces formes comme réellement « fautives », on ne peut donc penser qu'ils ironisent quant à l'évaluation enseignante. L'abondance des réponses du même type exclut aussi un biais du questionnaire qui serait de répondre « pour faire plaisir à l'enseignant ». Les entretiens montrent que « la déviance », fréquente, est assortie d'une sanction sociale : celui qui fait la faute se fait comprendre, mais on lui fait remarquer qu'il ne parle pas correctement ${ }^{19}$. Ces pratiques sont évoquées dans 21 entretiens sur 29 mais sont interprétées de façon différente. L'ensemble des réponses données permet de révéler la pluralité des positionnements et des stratégies. En stigmatisant par le rire, on permet à l'autre de se corriger. L'autre est même tenu d'accepter la sanction sous peine d'être exclu du groupe :

E d'accord / et alors euh comment ça s'passe quand un élève fait une faute de français entre vous?

17. Question posée dans la partie « toi et l'apprentissage » (B-6) : «Donne trois exemples de «fautes » en français qu'il ne faut pas faire entre élèves dans la cour ».

18. Par exemple : «prête-moi ton ball »; « je suis parti dans Langano »; « je suis dormi »; « va la demander».

19. Ces données n'excluent pas les cas dans lesquels « on ne se comprend pas », mais ils ne sont presque jamais évoqués par les élèves. 
ELISABETH (rire) on se moque de lui toute l'année!

$\mathrm{E}$ toute l'année?! (sourire) / le pauvre!

ELISABETH ben pas toute l'année mais + des semaines et des semaines ! tu fais une faute baka ${ }^{20}$ ! (rires) [...]

$\mathrm{E} \quad$ ça fait rire tout l'monde en fait /

ELISABETH oui!

Tous ou presque se présentent aussi comme ayant déjà fait des erreurs face aux autres. Certains acceptent la sanction et l'estiment bonne :

BRUKTAWIT [...] moi quand je fais une faute on me corrige / par exemple parfois j'confonds le féminin et le masculin /

E et quand les autres te corrigent comment tu réagis?

BRUKTAWIT c'est bien pour moi / (sourire).

D'autres condamnent la moquerie parce qu'elle provoque un fort sentiment de honte chez celui qui est repris :

$\mathrm{E} \quad$ et comment les élèves y réagissent?

GABRIEL ils rigolent / ils se moquent un peu / et après ils corrigent les fautes /

$\mathrm{E} \quad$ et alors la personne qui fait la faute comment elle réagit?

GABRIEL / ++ ils ont honte / quelquefois ils disent « j'ai fait exprès » pour avoir pas tellement honte /

E après est-ce que ça les aide à moins faire la faute?

GABRIEL oui /.

La moquerie est cependant toujours présentée comme une aide pour corriger sa faute. Pour Leo, on ne peut faire autrement qu'accepter la honte pour pouvoir se corriger.

E alors quand tu entends ces élèves dire ça comment tu réagis ou les autres comment ils réagissent?

LEO yen a qui rigolent et yen a d'autres qui corrigent et pis c'est tout //

E et ceux qui font les fautes comment ils réagissent?

LEO ils sont énervés /

$\mathrm{E} \quad$ pourquoi ils sont énervés?

LEO j'chais pas / ils ont honte aussi /

$\mathrm{E} \quad$ mais ils ont honte à quel moment?

20. Traduction littérale : «ça suffit ! » 
LEO quand on se moque d'eux /

E donc ils sont énervés parce qu'on se moque d'eux?

LEO non ils sont énervés aussi parce qu'ils font des fautes /

E mais ils s'en rendent compte quand ils font des fautes?

LEO non / si tu lui dis pas ils le savent pas /

E donc ils sont énervés quand on les corrige / et quand on se moque d'eux/ et alors qu'est-ce qu'il faudrait faire à ton avis?

LEO corriger!

E même si ça les énerve ?

LEO oui /

$\mathrm{E} \quad$ pourquoi?

LEO parce que c'est avec des erreurs qu'on peut réussir / alors que si on fait pas d'erreur je sais pas comment on peut progresser //.

Aïda, quant à elle, révèle que l'on ne sanctionne pas le chef du groupe, mais aussi que les «plus petits » peuvent revendiquer la norme endogène pour se défendre :

AÏDA ça dépend de quel élève que c'est si + si c'est le chef du groupe euh + personne ne lui + mais quand c'est un petit + on rigole et le plus souvent on essaye de le corriger / mais le plus souvent l'élève n'apprécie pas / qu'on essaye de le corriger en français //

E / comment qu'est-ce que comment ils ressentent ça à ton avis / pourquoi ils apprécient pas?

AÏDA parce que / quand / quand par exemple moi j'essaye de corriger il me dit « bon c'est pas grave t'as compris c'que j' voulais dire c'est l'essentiel».

Face à ces comportements récurrents, une solution « facile » (stratégie d'évitement) est de ne pas parler français, comme l'évoquent deux élèves. On parle alors amharique et/ou anglais. Les élèves pour qui la sanction interne est très forte ne parlent pas français en dehors des cours. L'insécurité peut conduire à stigmatiser celui qui utilise le français, lui reprochant d'utiliser la langue du pouvoir : « ah oui t'essayes de faire ce qu'on te dit $^{21}$ ! »

Nombre de «fautes » ne seraient pas corrigées parce qu'elles ne seraient pas remarquées par le plus grand nombre, comme le relèvent

21. Kidus. 
trois élèves ( « des fois ils les corrigent / des fois ils savent pas qu'ils ont fait des fautes $\left.{ }^{22} »\right)$. Par ailleurs, certaines « fautes » stigmatisées par le groupe n'en sont pas selon la norme française. Ainsi Ousmane raconte qu'on a sanctionné « je le lui ai dit», repris en « je lui ai dit ». Il a accepté la sanction sans douter de la validité de la correction, mais les moqueries qui en découlent sont pourtant très mal vécues : «oh ça fait mal ${ }^{23}$ ! »

La norme prescriptive est utilisée comme un produit social qui participe aux relations de pouvoir à l'intérieur de réseaux. Comme l'attestent Mariam, Aïda et Ousmane, plus on se sent proche de la personne «qui fait une faute » et plus on différencie la correction de la moquerie. S'opposant à ces humiliations, c'est l'argument de solidarité ethnique qui est ici convoqué par Michael, élève franco-éthiopien récemment arrivé au LGM :

MICHAEL : ben euh + par exemple quand ya des Éthiopiens qui ont du mal à parler français ils font des fautes par exemple / moi je sais que comme j'suis à moitié éthiopien et le fait que j'comprenne un peu leur milieu parce que j'me mélange un peu à eux / ben j'comprends que ils fassent des fautes par exemple « le maison » pour « la maison » avant j'rigolais aussi comme j'étais pas d'leur milieu / mais après des gens qui parlent français se sentent supérieurs parce qu'ils parlent français, les Français rigolent quand les Éthiopiens font des fautes et moi maintenant j'ai commencé à comprendre les Africains on est tous africains mais j'vais dire les Maliens tout ça / comme ils se mélangent pas avec eux ils rigolent et après quand ils ont un conflit avec cette personne ils disent « ouhai mais toi tu fais des fautes » tout ça /

Ces processus interactionnels correctifs existent aussi dans d'autres contextes ayant une configuration diglossique et l'adolescence favorise ce type d'attitudes, en particulier à l'école. Il est remarquable que cela n'empêche ici ni le parler mixte ni les erreurs fossiles, ni même la forte valeur associée à une identité d'élève du LGM fondée sur la diversité, la tolérance et l'unité. Pourtant, à cette valeur s'en associe une autre : celle de la distinction par le français.

22. Ashenafi.

23. Ousmane. 


\section{Une approche complexe des processus normatifs plurilingues}

L'hégémonie du français normé est réelle puisque tous s'accordent à dire qu'il est nécessaire de corriger les « fautes », même au prix d'une forte insécurité linguistique. Mais ces processus hégémoniques ne sont pas tant liés au code lui-même qu'à son statut de langue dominante dans l'établissement. Contrairement aux schémas classiques d'une diglossie vue comme séparant clairement deux variétés, on perçoit ici que les attitudes diffèrent selon que l'on donne la priorité à la distinction à l'intérieur de la communauté (ne pas être sanctionné, pouvoir même sanctionner) ou la distinction à l'intérieur de l'établissement (se dire adolescent, le dire autrement qu'en français). Associé à « un plurilinguisme de tolérance » au LGM, la focalisation sur ce qui serait des règles constitutives du français permet la distinction et, dans le même temps, renforce le sentiment d'une identité plurielle qui conjugue rôle d'élève, d'apprenant et d'adolescent. Mais on l'a vu, c'est la thématisation de ces règles qui a un pouvoir de distinction, sans inférer pour autant de leur acceptabilité pour un locuteur natif. La notion de « communauté linguistique », si elle est utile et nécessaire au chercheur en tant qu'outil heuristique, demande donc à être pensée en tant qu'objet complexe (Morin, 1990), selon un principe dialogique (qui intègre les paradoxes apparents entre deux observables), un principe de récursivité (et non de causalité linéaire entre pratiques et représentations langagières), et un principe hologrammatique qui affirme que le tout (ici les pratiques à l'échelle de la communauté perçues à un moment donné dans une situation donnée, et non dans leur ensemble) renvoie à ses parties (les différences individuelles de chaque membre), qui elles-mêmes renvoient au tout (les dynamiques langagières les plus diffusées dans le groupe).

Cette étude gagnerait à être poursuivie à propos des deux autres langues principales utilisées (l'amharique et l'anglais) et à être comparée à d'autres terrains plurilingues (scolaires ou non) de façon à permettre d'identifier la vitalité des processus normatifs à l'œuvre selon les contextes linguistiques. Mais parce qu'il s'agit d'un groupe d'élèves plurilingues, il a été possible d'identifier des enjeux normatifs non directement associés aux règles constitutives des codes linguistiques utilisés. Dans un milieu endolingue, la difficulté à catégoriser les variétés du français (Gadet, 2003) rend périlleuse la différenciation entre les normes actualisées dans les interactions et celles thématisées dans les discours. Entre le dire et le dit se tissent les enjeux identitaires des locuteurs. 
Les terrains plurilingues font donc réinterroger les catégorisations pertinentes pour une sociolinguistique qui vise à « savoir pourquoi, dans la relation au monde que peut entretenir un sujet ou un groupe, [la langue] est construite, interprétée comme, selon le cas, homogène ou inverse » (Robillard, 2008 : 35).

\section{ÉLÈVES CITÉS (TOUS PLURILINGUES)}

Aïda, nigérienne, est en seconde.

Ashenafi, éthiopien, est en sixième.

Bruktawit, éthiopienne, est en quatrième.

Elisabeth, italo-éthiopienne, est en seconde.

Gabriel, congolais, est en quatrième.

Kidus, éthiopien, est en première.

Leo, burundais, est en quatrième.

Mariam, éthio-malgache, est en quatrième.

Michael, franco-éthiopien, est en quatrième.

Ousmane, sénégalais, est en seconde.

\section{RÉFÉRENCES BIBLIOGRAPHIQUES}

Bavoux C., Prudent L.-J. et Warthon S. (2008) : Normes endogènes et plurilinguisme, Aires francophones, aires créoles, Paris, ENS Éditions.

BouRdieu P. (1982) : Ce que parler veut dire, L'économie des échanges linguistiques, Paris, Fayard.

BOYER H. (dir.) (2010) : Hybrides linguistiques, Genèses, statuts, fonctionnements, Paris, L'Harmattan.

CALVET L.-J. (1994) : Les voix de la ville, introduction à la sociolinguistique urbaine, Paris, Payot.

Demeulenaere P. (2003) : Les normes sociales, entre accords et désaccords, Paris, PUF.

GADET F. (2003) : La variation sociale en français, Paris, Ophrys.

Heller M. (2003) : Éléments d'une sociolinguistique critique, Paris, Didier.

LABOv W. (1976) : Sociolinguistique, Paris, Éditions de Minuit.

Marcellesi J.-B. (dir.) (1986) : Glottopolitique, Langages, nº 83.

Marcellesi J.-B., Bulot T. et Blanchet P. (2003) : Sociolinguistique, épistémologie, langues régionales, polynomie, Paris, L'Harmattan. 
Miguel Addisu V. (2010) : Apprendre en français au Lycée franco-éthiopien d'Addis-Abeba : une approche sociolinguistique à des fins didactiques, thèse de doctorat, université de Rouen.

Moore D. et Py B. (2008) : «Introduction : discours sur les langues et représentations sociales » dans G. Zarate, D. Lévy et C. Kramsch, Précis du plurilinguisme et du pluriculturalisme, Paris, Éditions des archives contemporaines, p. 271-279.

Moreau M.-L. (2000) : «La pluralité des normes dans la francophonie» dans La coexistence des langues dans l'espace francophone. Approche macrosociolinguistique, Paris, AUPELF-UREF, p. 137-151.

Moreau, M.-L. (éd.) (1997) : Sociolinguistique, concepts de base, Liège, Mardaga.

Morin E. (1990) : Communication et complexité, introduction à la pensée complexe, Paris, ESF.

Nicolaï R. (2011) : La construction du sémiotique, Sur les dynamiques langagières et l'activisme des acteurs de la communication, Paris, L'Harmattan.

Pöll B. et Schafroth E. (dir.) (2009) : Normes et hybridation linguistiques en francophonie, Paris, L'Harmattan.

Poutignat P. et Streiff-renart J. (2005) [1995] : Théories de l'ethnicité, Paris, PUF.

RAMTPON B. (2004) [1995] : Crossing : Language and Ethnicity among Adolescents, Manchester, St. Jerome Press.

Rey A. (1972) : «Usages, jugements et prescriptions linguistiques », Langue française, $\mathrm{n}^{\circ} 16, \mathrm{p} .4-28$.

- (2010) : «Paradoxe du français : une langue "victime" et "bourreau" » dans M. Abécassis et G. Ledegen (éds), Les voix des Français à travers l'histoire, l'école et la presse, vol. 1, Berne, Peter Lang, p. 111-121.

RoBILlard De D. (2008) : Perspectives alterlinguistiques, vol. 2 : Ornythorynques, Paris, L'Harmattan.

SEARLE J. R. (1972) : Les actes de langage, essai de philosophie du langage, Paris, Hermann.

ZRIBI-HertZ A. (2011) : « Pour un modèle diglossique de description du français : quelques implications théoriques, didactiques et méthodologiques », Journal of French Language Studies, vol. 21/2, p. 231-256. 
